\title{
Evaluación de la sustentabilidad ambiental de tres sistemas de producción agropecuarios, en el corregimiento Bolo San Isidro, Palmira (Valle del Cauca)
}

\author{
Reinaldo Giraldo Díaz \& Francis Liliana Valencia T. \\ reinaldo.giraldo@unad.edu.co, francis.valencia@unad.edu.co \\ Universidad Nacional Abierta y a Distancia (UNAD) \\ Escuela de Ciencias Agrícolas, Pecuarias y del Medio Ambiente (ECAPMA) \\ CEAD Palmira, Colombia
}

Resumen.- Este estudio gira en torno a la Evaluación de la Sustentabilidad Ambiental en tres sistemas de producción agropecuarios: Convencional, Agroecológico y en Transición a Agroecológico. El Sistema Convencional es un sistema que utiliza insumos químicos como herbicidas, insecticidas y pesticidas, maquinaria pesada como tractores y riego, y se enfatiza en la agricultura como negocio. El Sistema de Producción Transición a Agroecológico se encuentra en un proceso de reconversión tecnológica de convencional a agroecológica. En el Sistema de Producción Agroecológico hay una interrelación hombre-naturaleza más fuerte. Los estudios de Evaluación de la Sustentabilidad de los sistemas de producción resultan ser una herramienta valiosa para determinar el impacto de las tecnologías agroecológicas e identificar los puntos débiles de los sistemas que ponen en riesgo su sustentabilidad a corto, mediano y largo plazo. Asimismo, permiten un monitoreo rápido y permanente dentro de los sistemas, lo que facilita su evaluación, su seguimiento y mejoramiento.

Para la evaluación de la sustentabilidad ambiental se adoptó la metodología conocida Marco para la Evaluación de Sistemas de Recursos Naturales incorporando Indicadores de Sustentabilidad (MESMIS). Los atributos evaluados fueron: productividad, adaptabilidad, resiliencia, autogestión, confiabilidad y estabilidad.

El sistema de producción agroecológico es ambientalmente más sustentable que los sistemas de producción convencional y en transición a agroecológico, pues, en este sistema de producción se enfatiza en la conservación de los recursos naturales, en la preservación de la biodiversidad, en el uso de insumos de origen biológico para el control de plagas y enfermedades y hay una interacción hombre-naturaleza que no está mediada por la agroproductividad, sino por el respeto de los ciclos, ritmos y tiempos de la naturaleza, configurando un paisaje biodiverso y rico en singularidades.

Palabras clave: Adaptabilidad, Autogestión, Productividad, Resilicencia, Confiabilidad, Sustentabilidad

\begin{abstract}
This study focuses on Assessment of Environmental Sustainability in Three systems of agricultural production: Conventional, Agroecological and Transition to Agroecological. The conventional system is a system that uses chemical supplies such as herbicides, insecticides and pesticides, heavy machinery such as tractors and irrigation, and emphasis on agriculture as a business. The Transition to Agroecology systems is in the process of conversion from conventional to agro-ecological technology. In the Agroecological farming systems there is a strong human-nature relationship. Studies of Sustainability of production systems are a valuable tool to determine the impact of agro-ecological technologies and identify weaknesses in systems that threaten their sustainability in the short, medium and long term. They allow fast and continuous monitoring within systems, which facilitates their assessment, monitoring and improvement.
\end{abstract}


For the assessment of environmental sustainability the methodology known as Framework for Assessment of Natural Resources Systems was adopted, incorporating sustainability indicators (MESMIS). The attributes evaluated were productivity, adaptability, resilience, self-management, reliability and stability.

The agro-ecological production system is environmentally more sustainable than conventional production and transition to agro-ecological systems, because this production system emphasizes the conservation of natural resources, preservation of biodiversity, the use of biological supplies for control of pests and diseases and there is an interaction between man and nature that is not mediated by agro-productivity but respect for the cycles, rhythms and rhythms of nature, forming a biologically diverse landscape and rich in singularities.

Keywords: Adaptability, Self Management, Productivity, Resiliency, Reliability, Sustainability

\section{Introducción}

Los métodos orgánicos pueden producir suficiente alimento para todos sin disminuir la calidad ambiental ni reducir los recursos naturales. En 1989 el Consejo Nacional de Investigación de los Estados Unidos de América describió estudios de caso de ocho sistemas de producción orgánicos que abarcaban un rango de sistemas mixtos de granos/ganado desde 400 acres en Ohio, hasta de 1.400 acres de uvas en California y Arizona. Los rendimientos en los sistemas de producción orgánicos fueron iguales o mejores que los promedios de rendimiento de los sistemas convencionales intensivos de los alrededores. Estos sistemas productivos pudieron sostener el rendimiento año tras año sin deteriorar el ambiente y sin usar insumos sintéticos costosos, lo cual favoreció también la vida en el suelo, el mejoramiento de la calidad del agua y el valor de los alimentos tanto nutricional como económica (Acevedo 2000).

En este sentido, la agroecología es vista como una herramienta fundamental para mejorar la calidad de vida de las familias campesinas mediante el ordenamiento y utilización del territorio, de acuerdo a sus potencialidades y limitantes, que orientada hacia un desarrollo sustentable, aporta instrumentos y herramientas que permitan a los campesinos valorar y reconocer especies tradicionales con algunas formas de utilización en procesos integrados dentro de la parcela, producir alimentos no contaminados, conservar la biodiversidad y los recursos naturales, rescatar los saberes populares y ancestrales, formular y ejecutar proyectos productivos alternativos e impulsar formas asociativas y organizativas propias para la autogestión comunitaria (Alemán et al. 2001). La construcción de esta alternativa productiva integra lo ambiental, político, económico, social, cultural y plantea enormes retos teóricos y éticos.

El incremento en la producción y la productividad agrícola, pueden contribuir al desarrollo económico a través de la provisión de alimentos, vista esta de dos formas se considera que el incremento en la oferta de alimentos mejoraría el nivel de vida de los pobres tanto rurales como urbanos, y si existe exportación de productos agrícolas, ésta podría ser fuente generadora de divisas, las cuales servirían para importar bienes de capital que se utilizarían en otros sectores. También el desarrollo económico se vería incrementado por el sector agrícola al haber transferencia de fuerza de trabajo de la agricultura a la industria, de la ampliación del mercado para los productos industriales al existir aumentos en los ingresos netos de la población agrícola (Barajas 2005).

La importancia de la concepción de la finca como unidad de producción nos lleva a reflexionar sobre el interés y objetivos que se presentan en la realización de un análisis; la entrevista y la observación de la estructura y función, nos darán una idea global del sistema. Ejemplo de ello 
lo tenemos en los tipos de cultivo o animales que se encuentran en las prácticas de manejo, la toma de decisiones y las salidas o productos que se venden. Se supone que sistemas de cultivo que están orientados más por un sentido económico agroempresarial, con alta productividad y eficiencia, tendrán un menor número de componentes o agroecosistemas en razón de la homogeneidad de los mismos y de las prácticas que les son consustanciales, es decir, son sistemas de cultivo monoculturales cuyas labores están casi estandarizadas, mientras que los sistemas de producción campesina estarán conformados por un sinnúmero de agroecosistemas con múltiples interacciones entre ellos (Malagón \& Praguer 2001).

La importancia del estudio de los agroecosistemas radica en que su investigación se enfatiza en algunas características especiales de producción (procesos y técnicas de transformación), las cuales incluyen respuestas biológicas y económicas de cultivos agrícolas a determinadas prácticas de manejo, como en el caso de agroecosistemas con componentes de cultivos, o de incrementos en las variables productivas y reproductivas en animales de interés zootécnico, derivados de los arreglos establecidos por el productor.

La seguridad alimentaria de una familia significa lograr la satisfacción de las necesidades alimentarias en cantidad y calidad suficientes. Los tres elementos claves para esta seguridad los constituyen el acceso, la disponibilidad y el buen uso de los alimentos.

De allí que la educación alimentaria es un elemento estratégico importante en cualquier programa que tenga como fin reforzar y/o alcanzar la seguridad alimentaria. Igualmente, la capacitación para una mayor y mejor utilización de los cultivos nativos incide en la recuperación de la agricultura andina, apoya la tarea para alcanzar una nutrición adecuada y fomenta el uso de una amplia variedad de especies alimenticias, lo que contribuye a la conservación de la biodiversidad.
En el presente trabajo se hace referencia al término sustentabilidad ambiental, es decir, al equilibrio dinámico en las relaciones sociedadnaturaleza; la sustentabilidad ambiental es el resultado de un conjunto de acciones que se realizan con una visión integral de los procesos de desarrollo, en perspectiva de largo plazo (Gomero \& Velásquez 2000).

Se parte de la realidad y del contexto en la cual se está, y teniendo en cuenta su historia, se proyecta al futuro, con propósitos que orientan el aprovechamiento equitativo de los recursos, la participación de los actores sociales, el uso y conservación de la biodiversidad de acuerdo a las posibilidades de corto, mediano y largo plazo. El concepto de sustentabilidad ambiental permite analizar las condiciones que hacen posible que las comunidades locales, a pesar de las difíciles condiciones políticas, sociales y económicas, puedan estrechar relaciones conviviales con la naturaleza. Martin O'Connor, ecosocialista neozelandés y reconocido ambientalista a nivel mundial, sugiere que la mirada agro productiva está adquiriendo una nueva modalidad en lo que denomina la fase ecológica, es decir, la naturaleza no es vista como una realidad externa a ser explotada por cualquier medio, como en la concepción predominante de la modernidad; ahora la naturaleza es vista como una fuente de valor en sí misma. Por tanto, "la dinámica productivista cambia de forma, de la acumulación y crecimiento con base en una realidad externa, a la conservación y autogestión de un sistema de naturaleza productivista cerrada sobre sí misma” (Arango 2004).

La metodología MESMIS evalúa las iniciativas campesinas hacia la sustentabilidad, la autonomía, la autosuficiencia y la soberanía alimentaria. Las investigaciones realizadas han demostrado que los sistemas de producción orgánicos, agroecológicos o alternativos (términos que usaremos indistintamente) pueden ser tan productivos como los convencionales, prescinden de los agroquímicos, consumen menos energía importada, conservan los recursos na- 
turales y mantienen con vida el suelo y el agua (Speelman et al. 2007).

El estudio realizado tuvo como objetivo general evaluar la sustentabilidad ambiental de tres sistemas de producción agropecuarios manejados bajo los enfoques: Convencional, Agroecológico y Transición al Agroecológico en el corregimiento del Bolo San Isidro. Como objetivos específicos, caracterizar los sistemas de producción agrícola evaluados, establecer comparaciones entre los atributos de sustentabilidad de cada uno de los sistemas de producción agropecuaria; y, por último, evaluar la contribución al mantenimiento de la agrobiodiversidad de los tres sistemas de producción agrícola.

\section{Materiales y métodos}

Para los propósitos de este estudio se han establecido tres sistemas alternativos de producción agropecuaria que son:

- Sistema de Producción Agroecológico: (Villa Bernarda), la cual cuenta con una extensión de 4,6 ha. La propietaria, quien después de intentar con el paquete de la agricultura convencional realiza una conversión tecnológica a Agroecología, propuesta de vida con la cual se identifica la agricultora y cuyo proceso lleva más de 15 años.

- Sistema de producción Convencional: (La Guatinera). El predio tiene un área de 6,7 ha, en este sistema se trabaja con químicos de síntesis industrial para la producción de cultivos de plátano y zapallo.

- Sistema de producción Transición a Agroecológico: el predio tiene un área 1,92 ha. Comenzó con la agricultura convencional y después de un proceso de capacitación iniciado por diversas instituciones como la Universidad Nacional de Colombia sede Palmira, el SENA y la CVC está llevando a cabo una reconversión tecnológica.

La metodología empleada en este trabajo fue la propuesta por el Marco para Evaluación de Sistemas de Manejo de Recursos Naturales incorporando Indicadores de Sustentabilidad (MESMIS) López \& Masera (2000). MESMIS cuenta con una serie de propiedades o atributos generales. Estos atributos servirán de guía para el análisis de los aspectos más relevantes en la evaluación de la sustentabilidad de los sistemas de manejo de recursos naturales (Fig. 1); asimismo, el conjunto de atributos debe cubrir íntegramente aquellos aspectos que tienen un efecto sobre el comportamiento de un sistema de manejo. A continuación se presentaran los atributos generales propuestos en el MESMIS.

- Productividad. Es la habilidad del agroecosistema para proveer el nivel requerido de bienes y servicios

- Estabilidad. Con este término nos referimos a la propiedad del sistema de tener un estado de equilibrio dinámico estable. Es decir, que se mantenga la productividad del sistema en un nivel no decreciente a lo largo del tiempo bajo condiciones promedio o normales

- Resiliencia. Es la capacidad de retornar al estado de equilibrio o mantener el potencial productivo después de que el sistema ha sufrido perturbaciones graves

- Confiabilidad. Se refiere a la capacidad del sistema de mantenerse en niveles cercanos al equilibrio ante perturbaciones normales del ambiente

- Adaptabilidad (o flexibilidad). Es la capacidad del sistema de encontrar nuevos niveles de equilibrio -es decir, de continuar siendo productivo- ante cambios de largo plazo en el ambiente

- Autodependencia (o autogestión, en términos sociales). Es la capacidad del sistema de regular y controlar sus interacciones con el exterior

Teniendo en cuenta los atributos básicos para la sustentabilidad, un sistema de manejo puede ser considerado sustentable cuando permite simultáneamente: 


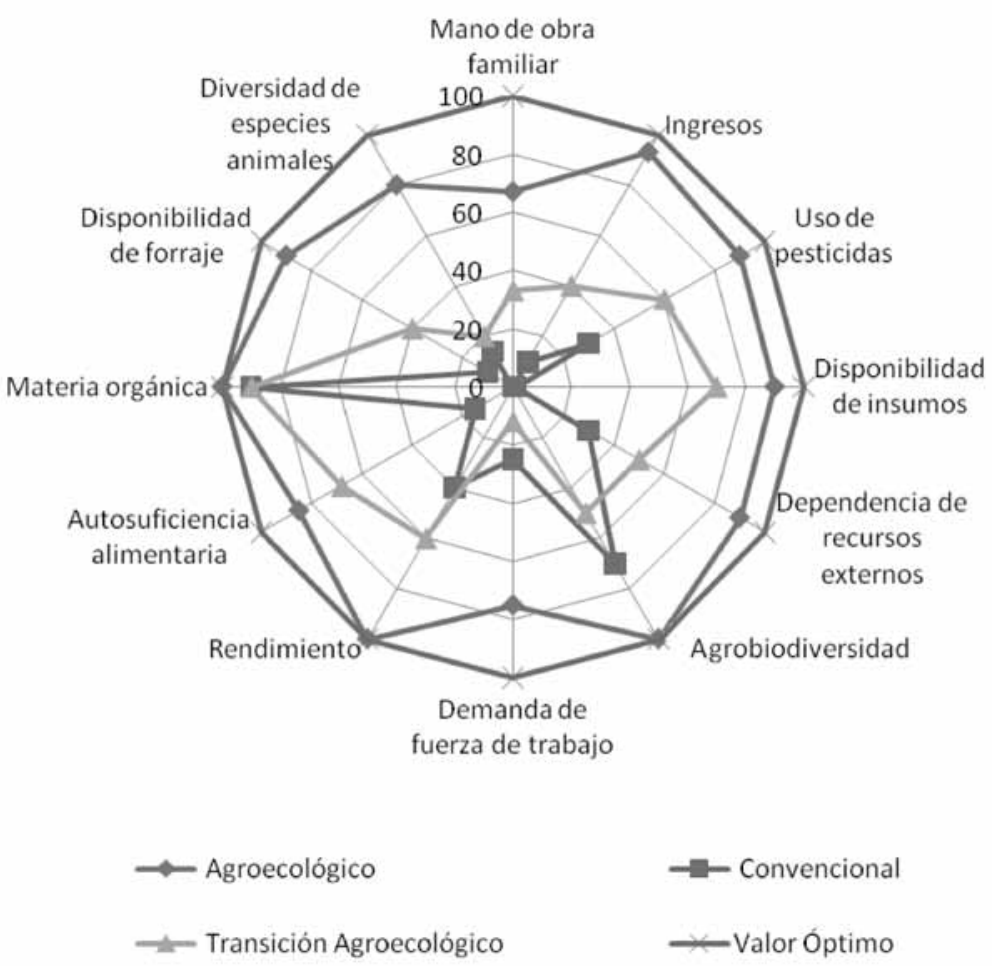

Figura 1. Diagrama de AMIBA para la comparación de indicadores de sustentabilidad en los tres distemas de producción (Agroecológico, Convencional y en Transición a Agroecológico) del corregimiento el Bolo San Isidro, Palmira, 2009.

- Conseguir un nivel alto de productividad mediante el uso eficiente y sinérgico de los recursos naturales y económicos

- Proporcionar una producción confiable, estable y resiliente, asegurando el acceso y disponibilidad de los recursos productivos, el uso renovable, la restauración y la protección de los recursos locales, una adecuada diversidad temporal y espacial del medio natural y de las actividades económicas

- Brindar flexibilidad (adaptabilidad) para amoldarse a nuevas condiciones del entorno económico y biofísico, por medio de procesos de innovación y aprendizaje

- Distribuir económicamente los costos y beneficios de los sistemas entre los agricultores, asegurando el acceso económico y la aceptación cultural de los sistemas propuestos
- Poseer un nivel aceptable de autodependencia (autogestión), para poder responder y controlar los cambios inducidos desde el exterior, manteniendo su identidad y sus valores (Muller 1996)

De acuerdo con esta metodología los pasos que se realizaron en esta investigación fueron:

\section{Definición del objeto de la evaluación}

Para evaluar este primer paso se tuvieron en cuenta tres actividades:

(a) Identificación del estudio de caso (corregimiento Bolo San Isidro-Palmira)

(b)Identificación de los sistemas de manejo a evaluar

(c) Caracterización del sistema de manejo de referencia (La Guatinera) que predomina 
en el corregimiento El Bolo San Isidro

(d) Caracterización de los dos sistemas alternativos: Agroecológico (Villa Bernarda) y Transición a Agroecológico

Para realizar esta caracterización de los sistemas de producción se hizo una descripción clara de:

- Los diferentes componentes biofísicos y microbiológicos de los sistemas (agua y suelo)

- Los insumos y productos necesarios (entradas y salidas) de los sistemas

- Las entradas y salidas de los sistemas y las relaciones entre sus diferentes componentes (pecuario y agrícola)

- Las prácticas agrícolas y pecuarias que involucran al Sistema de Producción Agroecológico

- Las principales características socioeconómicas y ambientales de los Agricultores

Identificación de los puntos críticos del sistema Se hizo un análisis de los aspectos o procesos que limitan o fortalecen la capacidad de los sistemas para sostenerse en el tiempo, para ello se hicieron entrevistas, reconocimiento del lugar y conteo de especies vegetales y animales. Una vez identificados los puntos críticos de los sistemas, se relacionaron con los diferentes atributos de sustentabilidad, con el fin de estar seguros de que la evaluación cubre todos los atributos.

\section{Selección de los criterios de diagnóstico e indicadores}

Se identificaron los diferentes criterios de diagnóstico e indicadores con el fin de evaluar el grado de sustentabilidad de los sistemas de manejo propuestos. Para ello se tuvo en cuenta que:

- Los criterios de diagnóstico se seleccionaron teniendo en cuenta que estos describan los atributos generales de sustentabilidad

- Los criterios seleccionados para la realización de este trabajo representan un nivel de análisis más detallado de estos, pero más general que los indicadores
- Constituyen el vínculo necesario entre atributos, puntos críticos e indicadores, con el fin de que estos últimos permitan evaluar de manera efectiva y coherente la sustentabilidad del sistema

- Los indicadores así estimados describen un proceso específico o un proceso de control

- Los indicadores aquí señalados dependen de las características del problema específico bajo estudio, de la escala de proyecto, del tipo de acceso y de la disponibilidad de datos

- Los indicadores propuestos son integradores, flexibles, fáciles de medir y entender, y adecuados al nivel de agregación del sistema bajo análisis

- Los indicadores se centran en aspectos claros y prácticos

- El conjunto de indicadores cubren tres dimensiones o áreas de evaluación: (a) social -incluyendo aspectos culturales y políticos(b) económica, (c) ambiental

\section{Medición y monitoreo de los indicadores}

Existe toda una gama de posibilidades para la medición de indicadores. Puesto que la sustentabilidad se refiere al comportamiento del sistema de manejo en el tiempo, se hizo énfasis en el análisis de series históricas o el modelaje de ciertas variables. Por ello, se seleccionaron los métodos más asequibles: (a) revisión bibliográfica, incluyendo información que permita establecer tendencias en el comportamiento de los indicadores; (b) mediciones directas: como análisis (agua y suelo); (c) entrevistas formales e informales con los agricultores y trabajadores de los tres sistemas de producción.

\section{Integración de resultados}

En esta etapa del ciclo de evaluación se resumieron e integraron los resultados obtenidos mediante el monitoreo de los indicadores. Es por lo tanto un momento clave en el ciclo de evaluación, pues se pasa de una fase de diferenciación centrada en la recopilación de datos para cada indicador, a otra de síntesis de la información, que preparará el camino para, posteriormente, poder emitir un juicio de valor sobre los sistemas de 
manejo analizados, que refleje como se comparan entre sí en cuanto a su sustentabilidad.

\section{Conclusiones y recomendaciones sobre los sis- temas de manejo}

En este último paso se recapitulan los resultados del análisis con el fin de emitir un juicio de valor para comparar entre sí a los distintos sistemas en cuanto a su sustentabilidad. Se plantean estrategias y recomendaciones que permitirán dar inicio al nuevo ciclo de evaluación de los sistemas de manejo en un estado cualitativamente diferente. Para esto último se realizó:

1. Una valoración de la sustentabilidad de los dos sistemas alternativos (Transitorio a Agroecológico y Agroecológico) en comparación con el sistema Convencional. La valoración es particular, pues "el sistema parece ser más sustentable en ciertos indicadores y atributos de sustentabilidad pero problemáticos o menos sustentable en otros".
2. Una discusión de los elementos principales que permiten o impiden a los sistemas alternativos mejorar la sustentabilidad con respecto al sistema de referencia. Dentro de este punto incluimos una discusión breve de los límites y oportunidades proporcionados por las condiciones de fronteras políticas, socioeconómicas o ambientales de los tres sistemas.

\section{Resultados}

La Evaluación de la Sustentabilidad Ambiental de los tres Sistemas de Producción (Tabla 1) muestra que el Sistema de Producción Agroecológico es ambientalmente más sustentable que los otros dos, el segundo en importancia en cuanto a sustentabilidad ambiental es el que se encuentra en Transición a Agroecológico, el que es a su vez más sustentable que el Sistema de Producción Convencional. Este orden no varía si la mirada se hace desde el punto de vista económico, es decir, si se valoran los sistemas

Tabla 1. Características de tres Sistemas de Producción Agropecuarios en El Bolo San Isidro Palmira-2009.

\begin{tabular}{|c|c|c|c|c|c|}
\hline & \multicolumn{2}{|c|}{$\begin{array}{l}\text { Determinantes del } \\
\text { agroecosistema }\end{array}$} & $\begin{array}{l}\text { Sistemas de Producción } \\
\text { Agroecologico }\end{array}$ & $\begin{array}{c}\text { Sistemas de } \\
\text { Producción } \\
\text { Convencional } \\
\end{array}$ & $\begin{array}{c}\text { Sistemas de Producción } \\
\text { en Transición en } \\
\text { Agroecológico } \\
\end{array}$ \\
\hline \multirow{8}{*}{ 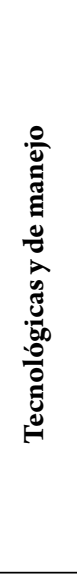 } & \multicolumn{2}{|c|}{$\begin{array}{l}\text { Tipo de especies y variedades } \\
\text { manejadas }\end{array}$} & Plátano, limón, mandarina y zapallo & Plátano, zapallo y maíz & $\begin{array}{l}\text { Plátano, limón, naranja, aguacate, } \\
\text { maíz y fríjol }\end{array}$ \\
\hline & \multicolumn{2}{|c|}{ Sistemas de cultivo } & Plátano asociado al aguacate y tomate & $\begin{array}{l}\text { Plátano asociado con } \\
\text { zapallo y maíz }\end{array}$ & Plátano asociado con maíz \\
\hline & \multicolumn{2}{|c|}{ Tecnología empleada } & $\begin{array}{l}\text { Uso de la agrodiversidad, riego y } \\
\text { labranza mínimas, técnicas de control } \\
\text { biológico de plagas y enfermedades }\end{array}$ & $\begin{array}{l}\text { Preparación de suelos } \\
\text { convencionales, énfasis } \\
\text { productivo en dos cultivos }\end{array}$ & $\begin{array}{l}\text { Uso de la agrodiversidad, riego } \\
\text { y labranza mínimas, técnicas de } \\
\text { control biológico de plagas y } \\
\text { enfermedades }\end{array}$ \\
\hline & \multicolumn{2}{|c|}{ Mano de obra empleada } & Baja & Media & Baja \\
\hline & \multirow{4}{*}{ 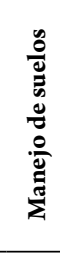 } & Fertilización & Abono orgánico & Triple 18 y supermagro & Abono orgánico \\
\hline & & $\begin{array}{c}\text { Practicas de } \\
\text { conservación }\end{array}$ & Barreras vivas, forraje, y abonos verdes & $\begin{array}{l}\text { Fertilizantes químicos y } \\
\text { algunos biopreparados }\end{array}$ & $\begin{array}{c}\text { Barreras vivas, forraje, y abonos } \\
\text { verdes }\end{array}$ \\
\hline & & $\begin{array}{l}\text { Manejo de plagas y } \\
\text { enfermedades }\end{array}$ & $\begin{array}{l}\text { Purines de aji, caldo de cenizas y } \\
\text { caldo bordeles }\end{array}$ & Lorsban & $\begin{array}{c}\text { Purines de ají, caldo de cenizas y } \\
\text { caldo bordeles }\end{array}$ \\
\hline & & Manejo de arvenses & Manual, pala y machete & $\begin{array}{l}\text { Guadaña, herbicidas, } \\
\text { roundup }\end{array}$ & Manual, pala y machete \\
\hline \multirow{2}{*}{ 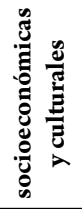 } & \multicolumn{2}{|c|}{$\begin{array}{c}\text { Características de los } \\
\text { productores }\end{array}$} & Produccion familiar & Producción comercial & Produccion familiar \\
\hline & $\mathrm{Ob}$ & etivo de la produccion & $\begin{array}{c}\text { Obtener ingresos monetarios, } \\
\text { autoconsumo, bienestar familiar } \\
\text { y conservación del sistema de } \\
\text { producción }\end{array}$ & $\begin{array}{l}\text { Obtener ingresos } \\
\text { monetarios }\end{array}$ & $\begin{array}{c}\text { Obtener ingresos monetarios, } \\
\text { autoconsumo, bienestar familiar } \\
\text { y conservación del sistema de } \\
\text { producción }\end{array}$ \\
\hline
\end{tabular}


productivos en relación con su rentabilidad, en este caso, el Sistema de Producción Agroecológico resulta ser el más rentable tanto por su variada oferta de productos como por la disponibilidad permanente de los mismos, lo que le genera un mayor ingreso; en segundo lugar se encuentra el Sistema de Producción en Transición a Agroecológico. El Sistema Convencional es el menos rentable económicamente debido a que sus gastos son mayores (Tabla 2).

Uno de los aspectos externos desfavorables para las prácticas de los agricultores tiene que ver con la producción de la caña de azúcar en el Bolo San Isidro; la fumigación impacta de manera fundamental el agua, el aire y el suelo, afectando de manera directa tanto la producción como la salud, el bienestar y la sustentabilidad ambiental de los tres sistemas de producción.

En cuanto a la seguridad alimentaria podemos decir que en el Sistema de Producción Agroecológico existe una mayor oferta de alimentos que conlleva a que pueda haber una mejor die- ta (teniendo en cuenta que de muchas de las especies se conocen su uso). En el Sistema de Producción en Transición a Agroecológico la oferta de productos alimenticios es menor debido a que el predio es más pequeño y a que el agricultor está adecuando el terreno. En el Sistema de Producción Convencional existe poca oferta de productos alimenticios ya que este sistema solo cuenta con tres cultivos.

La mano de obra familiar en el Sistema de Producción Agroecológico es mayor. Cada uno de los miembros del grupo familiar desempeña una labor; así, por ejemplo, una persona se encarga de las plantas ornamentales y medicinales cercanas a la vivienda; la propietaria quien es la coordinadora del Sistema de Producción, vela por la administración y mantenimiento de los subsistemas, como son los galpones, el ganado, las especies menores y los cultivos, por otra parte, otras personas colaboran indistintamente en algunas de las tareas del sistema de producción, se encargan de ayudar a mejorar técnicamente algunos de los procesos de agroin-

Tabla 2. Definición de valores óptimos y cálculos de valores ponderados de los indicadores de sustentabilidad.

\begin{tabular}{|c|c|c|c|c|}
\hline Indicador & Valor óptimo & $\begin{array}{c}\text { Sistema de } \\
\text { Producción } \\
\text { Agroecológico (\%) }\end{array}$ & $\begin{array}{c}\text { Sistema de } \\
\text { Producción } \\
\text { Convencional (\%) }\end{array}$ & $\begin{array}{c}\text { Sistema de Producción } \\
\text { en Transición a } \\
\text { Agroecológico }(\%)\end{array}$ \\
\hline Mano de obra familiar & $\begin{array}{c}100 \%=6 \\
\text { (personas/ha) }\end{array}$ & 67 & 0 & 33 \\
\hline Ingresos & $\$ 5.000 .000$ de Ingreso Neto & 93 & 10 & 40 \\
\hline Uso de pesticidas & $0 \%$ de dependencia & 90 & 30 & 60 \\
\hline $\begin{array}{l}\text { Disponibilidad de } \\
\text { insumos }\end{array}$ & $\begin{array}{l}0 \% \text { de requerimiento uso } \\
\text { externos }\end{array}$ & 90 & 1 & 70 \\
\hline $\begin{array}{l}\text { Dependencia de recursos } \\
\text { externos }\end{array}$ & $\begin{array}{l}0 \% \text { de dependencia con } \\
\text { respecto a recursos externos }\end{array}$ & 90 & 30 & 50 \\
\hline Agrobiodiversidad & Valor máximo posible & 100 & 70 & 50 \\
\hline $\begin{array}{l}\text { Demanda de fuerza } \\
\text { de trabajo (familiar y } \\
\text { externa) }\end{array}$ & $\begin{array}{c}100 \%=8 \text { (trabajadores } / \\
\text { ha-año) }\end{array}$ & 75 & 25 & 12 \\
\hline Rendimiento & $100 \%$ biomasa producida & 100 & 40 & 60 \\
\hline $\begin{array}{l}\text { Autosuficiencia } \\
\text { alimentaria }\end{array}$ & Valor máximo posible & 85 & 15 & 68 \\
\hline Materia orgánica & Mayor de 4 muy alto & 100 & 90 & 90 \\
\hline Disponibilidad de forraje & $\begin{array}{c}\text { Valor del sistema } \\
\text { autosuficiente en rastrojo }\end{array}$ & 90 & 10 & 40 \\
\hline $\begin{array}{l}\text { Diversidad de especies } \\
\text { animales }\end{array}$ & Valor de la localidad 15 & 80 & 14 & 20 \\
\hline
\end{tabular}


dustria artesanal como son la elaboración de quesos, yogurt, mermeladas y embutidos. En el Sistema de Producción Convencional no existe mano de obra familiar ya que este cuenta con dos trabajadores, encargados de manejar y velar por la productividad del sistema. En el Sistema de Producción en Transición a Agroecológico la mano de obra es menor ya que solo cuenta con la colaboración del propietario y su esposa.

La autosuficiencia alimentaria en el Sistema de Producción Agroecológico es del (85\%); el sistema cuenta con una diversidad de alimentos tanto vegetal como animal y sus productos son orgánicos; también cuenta con un banco de plantas medicinales, en el Sistema Convencional la autosuficiencia alimentaria es del (15\%); este sistema productivo se dedica al plátano con fines de comercialización; en el sistema en Transición a Agroecológico la autosuficiencia agropecuaria es del (68\%); los excedentes son mínimos pero la oferta alimentaria es adecuada y cuenta con proteína animal y vegetal.

La materia orgánica en los dos sistemas productivos (Convencional y en Transición a Agroecológico) es aceptable (Tabla 1), en el Sistema Agroecológico es del (100\%). El contenido de materia orgánica es esencial para la fertilidad y la buena producción agropecuaria. Los suelos sin materia orgánica son suelos pobres y de características físicas inadecuadas para el crecimiento de las plantas. De allí la importancia de este resultado tanto para la fertilidad del suelo como para la valoración de la calidad nutricional de los productos. En el Sistema de Producción Agroecológico se reciclan todos los materiales de los subsistemas. Teniendo en cuenta que cualquier residuo vegetal o animal es materia orgánica, y su descomposición los transforma en materiales importantes en la composición del suelo y en la producción de plantas, se resalta que su descomposición por microorganismos y transformación en materia adecuada para el crecimiento de las plantas, conocida como humus, genera una mayor disponibilidad de nutrientes, haciendo el sistema productivo independiente de los nutrientes externos.
En cuanto a la diversidad de especies animales es mayor en el Sistema Agroecológico, pues presenta una variedad de animales, la alimentación de estos animales se hace con insumos de los subsistemas. En el Sistema Convencional hay presencia de especies animales para el consumo. En el Sistema en Transición a Agroecológico cuenta con poca especies entre ellas gallinas, patos, perros y conejos.

La disponibilidad de forraje es mayor en el Sistema de Producción Agroecológico, del orden del (90\%). En segundo lugar se encuentra el Sistema de Producción en Transición a Agroecológico que es del (40\%). Por último, tenemos al Sistema Convencional, con un (10\%).

\section{Discusión y conclusiones}

El sistema de producción agroecológico es ambientalmente más sustentable que los sistemas de producción convencional y en transición a agroecológico, pues, en este sistema de producción se enfatiza en la conservación de los recursos naturales, en la preservación de la biodiversidad, en el uso de insumos de origen biológico para el control de plagas y enfermedades y hay una interacción hombre-naturaleza que no está mediada por la agroproductividad, sino por el respeto de los ciclos, ritmos y tiempos de la naturaleza, configurando un paisaje biodiverso y rico en singularidades.

El sistema de producción agroecológico es más sustentable económicamente que los sistemas de producción convencional y en transición a agroecológico, debido a la amplia oferta de productos para el mercado y a la calidad de los mismos, por lo cual los precios son más altos. Los Sistemas de producción Agroecológico y en Transición a agroecológico cuentan con mayor mano de obra tanto externa y familiar que el sistema de producción convencional, donde se genera poca demanda de fuerza de trabajo y es de carácter externo. Condiciones similares evidencia (Cárdenas et al. 2006), ante el entorno global y las políticas macroeconómicas, 
que se han impuesto condiciones sumamente desfavorables para la economía campesina; ha surgido formas alternativas de producción y comercialización agropecuaria que permitan la sustentabilidad de la producción, el mejoramiento de las condiciones de vida de los campesinos y la conservación de los recursos naturales. Algunas de estas formas alternativas que brindan soluciones a la problemática productiva con tecnologías agroecológicas se desconocen muchos de los impactos que pueden tener dentro de los sistemas campesinos.

Para los agricultores que están interesados en realizar una reconversión tecnológica de sistemas de producción agroproductivistas a agroecológicos resulta de particular importancia resaltar que el cambio es viable tanto económico, social y ambientalmente. Los estudios de Evaluación de la Sustentabilidad de los sistemas de producción resultan ser una herramienta valiosa para determinar el impacto de las tecnologías agroecológicas e identificar los puntos débiles de los sistemas que ponen en riesgo su sustentabilidad a corto, mediano y largo plazo (CIAT 1998). Asimismo, permiten un monitoreo rápido $\mathrm{y}$ permanente dentro de los sistemas, lo que facilita su evaluación, su seguimiento y mejoramiento. El Sistema de Producción Convencional tiende a ser desplazado por el Sistema de Producción Agroecológico debido a las ventajas que este ofrece.

Como principales recomendaciones, resulta conveniente la socialización con campesinos de la región que pueden estar en condiciones similares respecto de los tres agroecosistemas analizados, es necesario que trabajos de esta naturaleza se realicen en otros lugares con el fin de crear un sistema de información que permita establecer criterios claros y precisos en cuanto a la evaluación y sostenibilidad de los distintos agroecosistemas considerados. En el análisis de agua (calidad de agua para riego y coliformes totales) se recomienda a la población del Bolo especialmente a las fa- milias de los tres sistemas de producción objeto de estudio que ésta no sea utilizada para consumo humano ni para riego de hortalizas, a menos que se haga un proceso de potabilización. En los Sistemas de Producción Agroecológico y Transición a Agroecológico le sugerimos a los agricultores adoptar medidas de control para evitar la contaminación del agua en los pozos (entre ellas adecuar los pozos colocándole una tapa para evitar el contacto con los animales). En el Sistema de Producción Agroecológico se requiere la construcción de un biodigestor para realizar un control de las emisiones de aguas residuales contaminadas con porquinaza y otros estiércoles. Se debe orientar a la comunidad del Bolo San Isidro sobre la buena utilización del uso del suelo (por ejemplo tecnología limpias como un Sistema Agroecológico) para evitar la contaminación del ambiente y mejorar la calidad de vida de los habitantes.

En el Sistema de Producción en Transición a Agroecológico el agricultor debe buscar fuentes de comercialización para sus productos, pues, los excedentes producidos se desaprovechan. Para el Sistema de Producción Convencional se recomienda la asociación de cultivos (por ejemplo frutales) y la adopción de productos orgánicos para mejorar la calidad del suelo, sus productos e ingresos.

\section{Literatura citada}

Acevedo, A. 2000. Agricultura sustentable en el Trópico: principios, estrategias y práctica. Armero Guayabal, Colombia.

Alemán, T., J. Nahed \& J. Lopez. 2001. Sustentabilidad y agricultura campesina: la producción agrosilvopastoril en los altos de Chiapas, México. LEISA, Revista de Agroecología, Edición especial. pp. 18-23.

Arango, H. 2004. Bases e instrumento de apoyo para la planificación participativa de fincas. En: Memorias Seminario Internacional: Manejo Soste- 
nible de Sistemas de Producción en los Andes con Énfasis en Ganadería. Universidad de Caldas, Manizales.

Cárdenas, G., H. Giraldo, A. Idárraga \& L. Vásquez. 2006. Desarrollo y validación de metodología para evaluar con indicadores la sustentabilidad de sistemas campesinos de la asociación de caficultores orgánicos de Colombia-ACOC. Boletín de Investigaciones Unisarc, 4(2): 22-46.

Castaño, G. 1993. El Manejo de la biodiversidad por parte de las comunidades campesinas de la región central del Valle del Cauca. CEREC, Bogotá.

CIAT, Centro Internacional de Agricultura Tropical. 1998. Indicadores de sustentabilidad: una visión para América Latina y el Caribe. Palmira.

Gomero, L. \& H. Velásquez. 2003. Evaluación de la sustentabilidad del sistema de algodón orgánico en la zona de Trópico Húmedo del Perú. LEISA, Revista de Agroecología, Ocho estudios de caso, Edición especial 19: 47-52.
López, S. \& O. Masera. 2000. (Ed.). Sustentabilidad y sistemas campesinos. Cinco experiencias de evaluación en el México rural. GIRA; MundiPrensa, PNUMA, México.

Malagón, M.R. \& M.M. Prager. 2001. El enfoque de sistemas: una opción para el análisis de las unidades de producción agrícola. Facultad de Agronomía, Universidad Nacional de Colombia. Palmira. 190 p.

Muller, S. 1996. Cómo medir la sustentabilidad. Una propuesta para área de la agricultura y de los recursos naturales. Serie de documentos sobre agricultura sustentable y recursos naturales. IICA; GTZ, San José de Costa Rica.

Speelman, E., S. López, N. Aliana, M. Astier \& O. Masera. 2007. Ten years of sustainability evaluation using the MESMIS framework: Lessons learned from its application in 28 Latin American case studies. International Journal of Sustainable Development \& World Ecology, 14(4): 345-361.

Recibido: 16 de agosto de 2010 Aceptado: 17 de noviembre de 2010 